\title{
Editorial
}

\section{What about HES in burn patients?}

\section{Evaluation of the actual evidence}

Following the 2013 alert by the European Medicines Agency (EMA) discouraging the use of hydroxyethyl-starch solutions (HES) in critically ill and burn patients [1], most specialized units treating burn victims have stopped using HES in this population. But, how well-founded was the EMA alert in this specific type of patient?

A major burn patient is defined as one with burn injury affecting at least $20 \%$ or $30 \%$ of the body surface area (BSA). At this percentage, the patient experiences a systemic impact, characterized by an inflammatory response with microvascular changes that lead to altered capillary permeability, and hypovolemic and cell shock, commonly known as burn shock [2]. It is recognized that intravenous fluid resuscitation is essential to improve survival in major burn patients, as they are in an exceptionally severe clinical state. In addition to the above-mentioned systemic changes, they may experience massive bleeding when undergoing surgical treatment for their injuries and are highly susceptible to the development of infection. But not all victims of burn injury are major burn patients, and not all develop sepsis or have the same systemic repercussions. The different etiologies and severity of the lesions makes this a very heterogeneous patient population, and it is surprising that the European Medicines Agency considers them all in the same manner with respect to HES administration.

We believe that the alert emitted, which explicitly says "Hydroxyethyl-starches (HES) should no longer be used in patients with sepsis, burn injuries or critically ill patients" [1] lacks specific information. Given that there is considerable variability in the characteristics of burn patients and that these require advanced fluid therapy management [2], data are needed on the severity of the injury, the affected BSA, systemic involvement, and the time during which this recommendation should be applied.

Several questions come to mind in relation to this recommendation: Should solar burns or $1 \%$ burns be taken into account in a trauma patient with bleeding? Is it only appropriate for major burns? How long should it be applied? During the first $24 \mathrm{~h}$, as is done with the remaining colloids in patients with burns? One week? One month? And what is quite important: on what basis did this specific recommendation for burn patients emerge?

When the alert was communicated, it was based on a series of large, recently published studies: 6S [3], CRYSTMAS [4], CHEST [5], CRISTAL [6], and VISEP [7]. But, were burn patients actually included in these studies? To answer our concerns about the current recommendations for these patients, we set out to review the methods used in these studies, and here we present our results.

The 6S study [3], involving 798 patients and excluding those with burns affecting $>10 \%$ BSA, compared mortality and acute kidney injury after initial resuscitation with Ringer's acetate (RA) versus HES 130/0.42. It is surprising that only burns involving $<10 \%$ BSA were accepted, when these patients are not usually considered critically ill and are unlikely to develop severe sepsis. The study concluded that patients with severe sepsis or septic shock receiving HES had a higher risk of death and renal replacement requirement at 90 days than those given RA. Of note, patients were randomized after the first $24 \mathrm{~h}$, once they had been stabilized and when they may already have received 1 liter of synthetic colloids, as in the majority of these studies $[3,5,8]$. Furthermore, 38 patients in the RA group received synthetic colloids without being excluded from the statistical analysis. As to the results, there were no significant differences in mortality at 28 days, but differences were seen at 90 days. Mortality was related to the requirement for renal replacement therapy (RRT), but the indications for this therapy were not defined in the study protocol and were left to the discretion of the attending physician. The number of patients with a definite RRT requirement was exactly the same in the two groups. There were no significant differences between the groups for the authors' defined measure of renal failure, for the urinary output (UO) values, or for patients with doubling of plasma creatinine levels. With regard to the RIFLE score, the raw data were given in an appendix, but statistical significance was not calculated. No significant differences were seen for the overall SOFA score, but the renal subscore data were not provided; instead, they were combined with those of patients undergoing RRT.

This manuscript was not screened for plagiarism. 


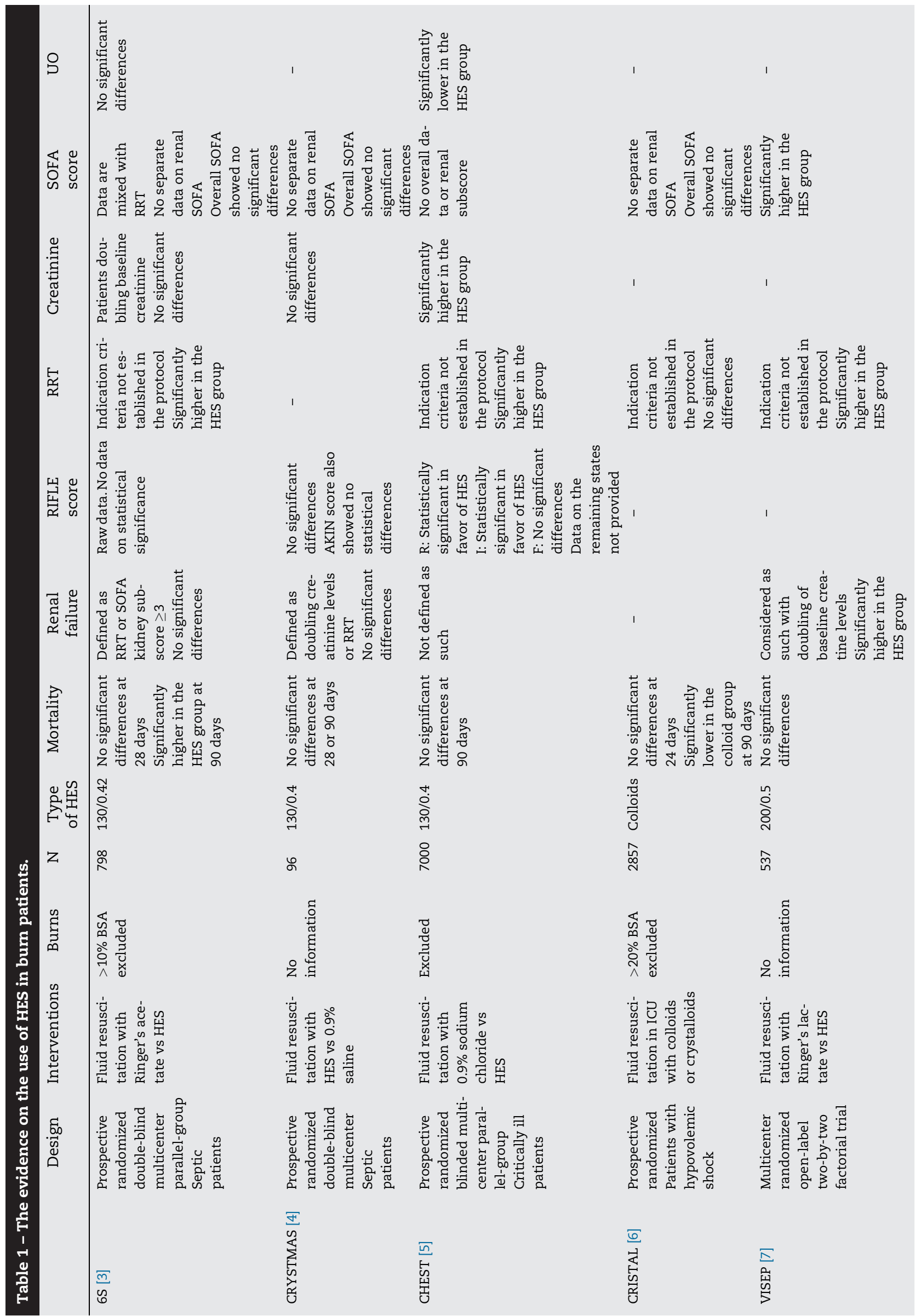




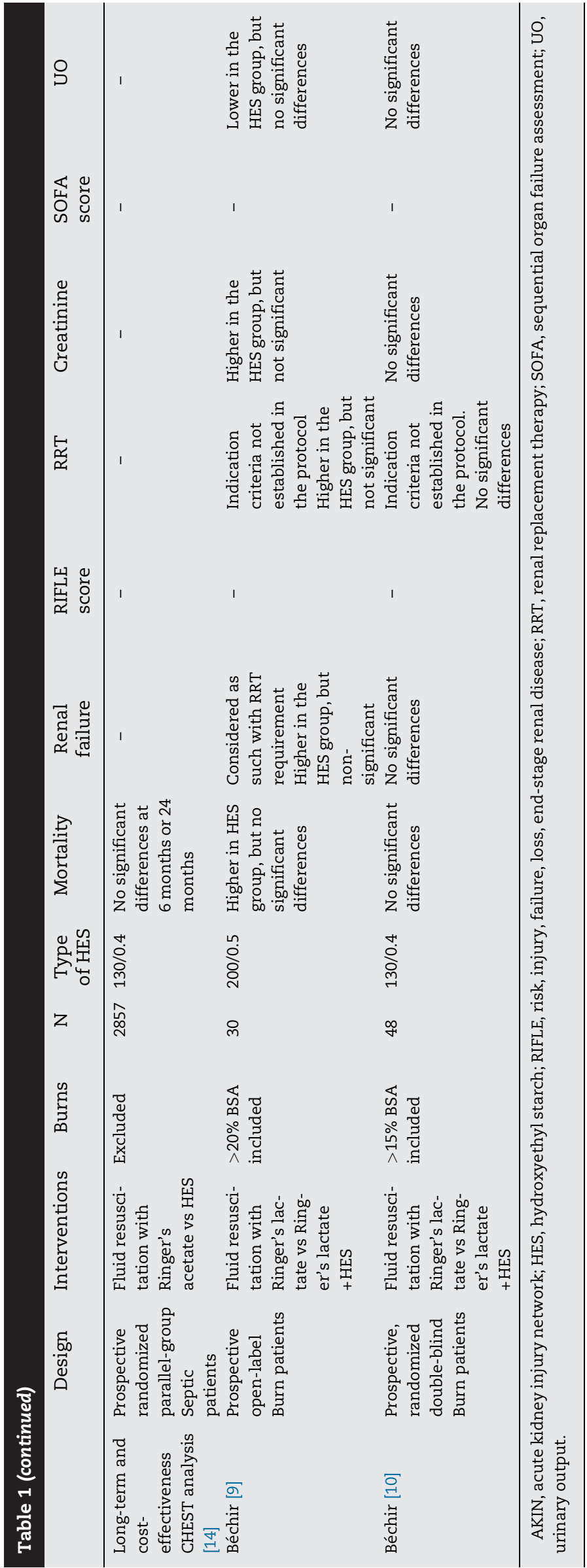


The multicenter CRYSTMAS [4] study, including 196 patients with severe sepsis, compared the volume of HES (130/ 0.4 ) versus $0.9 \%$ saline needed to hemodynamically stabilize patients, and concluded that a significantly lower volume of HES was infused on the first day. There were no significant differences in 28- and 90-days mortality, volume infused in the first 4 days, hemostatic changes, or incidence of acute kidney injury. Furthermore, urinary biomarkers showed no significant differences regarding tubular or glomerular involvement. There was no information on whether burn patients were included.

CHEST [5], another important multicenter study, analyzed 7000 patients, and burns were considered an exclusion criterion. HES (130/0.4) was compared with $0.9 \%$ saline for resuscitation in intensive care patients. In this study HES was associated with a higher incidence of renal replacement therapy as indicated by the attending doctor, although there were no significant differences in the severity of acute kidney injury estimated by the RIFLE score, and more patients were classified in the risk and injury stage in the saline group. No significant differences were found for 90-days mortality, ICU stay, or hospital stay. Creatinine concentration and UO were significantly higher in patients receiving saline, but in the HES group creatinine values were around $100 \mu \mathrm{mol} / \mathrm{L}$ and diuresis $1500 \mathrm{~mL} /$ day; hence the differences would seem to have little clinical relevance.

In CRISTAL [6], a multicenter study including 2857 patients, burn victims with $>20 \%$ BSA involvement were excluded. Resuscitation with colloids (HES, dextrans, gelatins, and albumin) or crystalloids (0.9\% saline, hypertonic solutions, and Ringer's lactate [RL]) was compared in intensive care patients in hypovolemic shock. There were no significant differences in mortality at 28 days, but the survival rate was higher in colloid-treated patients at 90 days. The colloid group remained more days without vasopressor therapy and had a lower incidence of mechanical ventilation. Of note, the study population was very heterogeneous and there were differences in mortality between centers. This study also provided separate data on mortality in HEStreated patients. This group also showed no significant differences with regard to mortality at 28 or 90 days except in the comparison of patients who received only one type of fluid. In this analysis, 90 days mortality was significantly lower in patients receiving HES than in those given isotonic saline solution.

As to the VISEP [7] study, it is important to note that the starch used was hypertonic pentastarch, a formula that differs completely from the fourth-generation isotonic starches currently used in Europe. Hence, as these are different products, the results obtained should not be extrapolated to other starches. VISEP was a randomized multicenter study including 537 patients with sepsis who underwent resuscitation with HES 200/0.5 (10\%) or RL. Mortality did not differ between the groups, but renal failure and RRT rates were significantly higher in the HES group. There was no mention of burn patients at any point in the article. Furthermore, the authors reported protocol violations and stated that the HES dose administered was $2400 \mathrm{~mL}$ in $24 \mathrm{~h}$, an amount almost double the maximum dose used in Europe.
Two studies specifically assessing HES use in burn patients are available, both by the same author $[9,10]$. The first, published in 2010 [9], included 30 patients and compared resuscitation using mixed therapy (crystalloids+HES 200/0.5 [10\%]) with RL-based therapy. Mortality and renal failure were somewhat higher in the HES group, but the results were not significant. The second study, published in 2013, was a randomized, double-blind study with 48 patients comparing resuscitation with RL or with mixed therapy (RL+HES 130/0.4 [6\%]). In this case there were no differences in terms of mortality, renal failure, or other associated morbidities between the two groups [10].

Following the HES alert, various authors have voiced concern about the methodology used in the studies upon which it was based [11,12], and several new studies and reviews on HES use in different types of patients have emerged. More than 20 new related studies are registered in Clinical Trials, and one of them, still to be published, is in burn patients [13]. In addition, a long-term, costeffectiveness study was carried out in a CHEST cohort in 2016 that found no differences in mortality or any of the other indicators evaluated at long term between the HES and $0.9 \%$ saline groups [14]. For our part, we conducted a retrospective study whose preliminary results were recently presented in the 2017 European Anaesthesiology Congress. The study, performed in major burn patients, found no significant differences in mortality at 28 or 90 days associated with administration of HES 130/0.4 versus other fluids [15].

The results of the analysis of these studies are shown in Table 1. It is surprising that only one study in 798 patients actually found a higher mortality rate in patients receiving HES 130/0.42 and only at 90 days, not at 28 days. Moreover, the authors reported a greater RRT requirement without specifying whether the indication was for renal failure. Finally, major burn patients were excluded from this study [3]. None of the available studies performed with HES 130/ 0.4 found differences in mortality in burn patients or critically ill patients.

As is well recognized, RRT use cannot be equated with end-stage renal failure and even less so if the criteria for starting RRT are not clearly established in the study protocol. Various reasons apart from renal failure can prompt the clinician to decide on RRT. We consider the lack of information on what constituted severe renal failure in these studies to be a limitation affecting the interpretation of the results.

This analysis of the current evidence regarding HES use in major burn patients leads us to conclude that despite the European Medicines Agency recommendations set down four years ago, to this day there is still no scientific evidence supporting a contraindication for last-generation HES use in patients with burn injuries. None of the related studies have shown an increase in mortality or renal failure in major burn patients treated with HES.

\section{Conflicts of interest}

All authors declare no conflicts of interest. 


\section{Authors contribution}

Patricia Guilabert helped design the study, conducted the study, analyzed the data, wrote the manuscript, and reviews.

Luis Abarca, Nuria Martin and Gemma Usua analyzed the data, and wrote the manuscript.

Juan P Barret helped final approval.

Maria J Colomina wrote the manuscript and reviews and helped final approval.

\section{Acknowledgement}

To Yolanda Diez, for teaching us everything she knew about the burn patient when we were just starting out.

R E F E R E N C E S

[1] European Medicines Agency. Hydroxyethyl-starch solutions (HES) should no longer be used in patients with sepsis or burn injuries or in critically ill patients - CMDh endorses PRAC recommendations HES will be available in restricted patient populations. Ema/640658/2013 2013; 44:1-3.

[2] Guilabert P, Usúa G, Martín N, Abarca L, BarretJP, Colomina MJ. Fluid resuscitation management in patients with burns: update. Br J Anaesth 2016;117:284-96.

[3] Perner A, Haase N, Guttormsen AB, Tenhunen J, Klemenzson G, Åneman A, et al. Hydroxyethyl starch 130/0.42 versus Ringer's acetate in severe sepsis. N Engl J Med 2012;367:124-34.

[4] Guidet B, Martinet O, Boulain T, Philippart F, Poussel JF, Maizel J. Assessment of hemodynamic efficacy and safety fluid replacement in patients with severe sepsis: the CRYSTMAS study. Crit Care 2012;16:R94.

[5] Myburgh JA, Finfer S, Bellomo R, Billot L, Cass A, Gattas D, et al Hydroxyethyl starch or saline for fluid resuscitation in intensive care. N Engl J Med 2012;367:1901-11.

[6] Annane D, Siami S, Jaber S, Martin C, Elatrous S, Declère AD et al. Effects of fluid resuscitation with colloids vs crystalloids on mortality in critically ill patients presenting with hypovolemic shock: the CRISTAL randomized trial. JAMA 2013;310:1809-17.

[7] Meier-hellmann A, Ragaller M, Weiler N, Moerer O, Gruendling $\mathrm{M}$, Oppert M, et al. Intensive insulin therapy and pentastarch resuscitation in severe sepsis. N Engl J Med 2008;358:125-39.

[8] Brunkhorst FM, Engel C, Bloos F, Meier-Hellmann A, Ragaller $\mathrm{M}$, Weiler $\mathrm{N}$, et al. Intensive insulin therapy and pentastarch resuscitation in severe sepsis. N Engl J Med 2008;358:125-39.
[9] Béchir M, Puhan MA, Neff SB, Guggenheim M, Wedler V, Stover $J F$, et al. Early fluid resuscitation with hyperoncotiv hydroxiethyl starch 200/0. 5 (10\%) in severe burn injury. Crit Care 2010;14:R123.

[10] Béchir M, Puhan MA, Fasshauer M, Schuepbach RA, Stocker R, Neff TA. Early fluid resuscitation with hydroxyethyl starch 130/0.4 (6\%) in severe burn injury: a randomized, controlled, double-blind clinical trial. Crit Care 2013;17(6):R299.

[11] Chappell D, Jacob M. Hydroxyethyl starch-the importance of being earnest. Scand J Trauma Resusc Emerg Med 2013;21:61.

[12] Chappell D, Jacob M. Twisting and ignoring facts on hydroxyethyl starch is not very helpful. Scand J Trauma Resusc Emerg Med 2013;21:85.

[13] Moiemen N. Fluid resuscitation in patients suffering from burns injury. Clin. trials identifier NCT01689506.

[14] Taylor C, Thompson K, Finfer S, Higgins A, Jan S, Li Q et al. Hydroxyethyl starch versus saline for resuscitation of patients in intensive care: long-term outcomes and cost-effectiveness analysis of a cohort from CHEST. Lancet Respir Med 2016;4:818-25

[15] Guilabert P, Usúa G, Abarca L, Nuria M, Karen S, Sharp A. The use of hydroxyethyl starch130/0.4 in major burns: a retrospective study. Eur J Anaesthesiol 2016;34(e-suppl):262.

Patricia Guilabert Luis Abarca

Nuria Martin

Gemma Usua

Anesthesia and Critical Care Department, University Hospital Vall d'Hebron, Barcelona, Spain

Juan P. Barret

Plastic Surgery Department and Burn Centre, University Hospital Vall d'Hebron, Barcelona, Spain

Maria J. Colomina Anesthesia and Critical Care Department, University Bellvitge Hospital, Barcelona, Spain

* Corresponding author at: University Hospital Vall d'Hebron, Area de traumatologia, Departamento de Anestesiologia y Reanimacion, Paseo de la vall d'Hebron 119-129, 08035 Barcelona, Spain. Fax: +34 934893403

E-mail address: patricia.guilabert@gmail.com (P. Guilabert).

Available online $\mathrm{xxx}$

http://dx.doi.org/10.1016/j.burns.2017.09.023 ๑) 2017 Elsevier Ltd and ISBI. All rights reserved. 